\title{
铜(I)催化条件下酮肟衍生物与苄烯丙二腈的环化反应构建 2-氨基吡啶
}

\author{
蔡忠建陆新谋汪顺义* 纪顺俊* \\ (苏州大学 材料与化学化工学部 苏州 215123)
}

\begin{abstract}
摘要 研究了铜催化条件下, 酮肜的衍生物与芐烯丙二腈的环化反应, 考察不同的催化剂、溶剂、添加剂等对反应的 影响，成功的构建了一系列的 2-氨基吡啶骨架化合物. 并对苯乙酮肟以及芳烯丙二腈芳环上不同位置具有不同性质取 代基的底物进行了拓展，以 $31 \% \sim 81 \%$ 的收率分离得到相应的目标产物，反应具有较好的底物普适性.
\end{abstract}

关键词 铜(I); 苯乙酮肜的衍生物; 芐烯丙二腈; 环化反应; 2-氨基吡啶

\begin{tabular}{|c|}
\hline $\begin{array}{l}\text { Copper-Catalyzed Cyclization of Oxime Acetates with } \\
\text { 2-Benzylidenemalononitriles for Synthesis of 2-Aminonicotinonitriles }\end{array}$ \\
\hline $\begin{array}{c}\text { Cai, Zhongjian } \quad \text { Lu, Xinmou } \quad \text { Wang, Shunyi* Ji, Shunjun* } \\
\text { (Key Laboratory of Organic Synthesis of Jiangsu Province, College of Chemistry, Chemical Engineering and Materials } \\
\text { Science, Soochow University, Suzhou 215123) }\end{array}$ \\
\hline 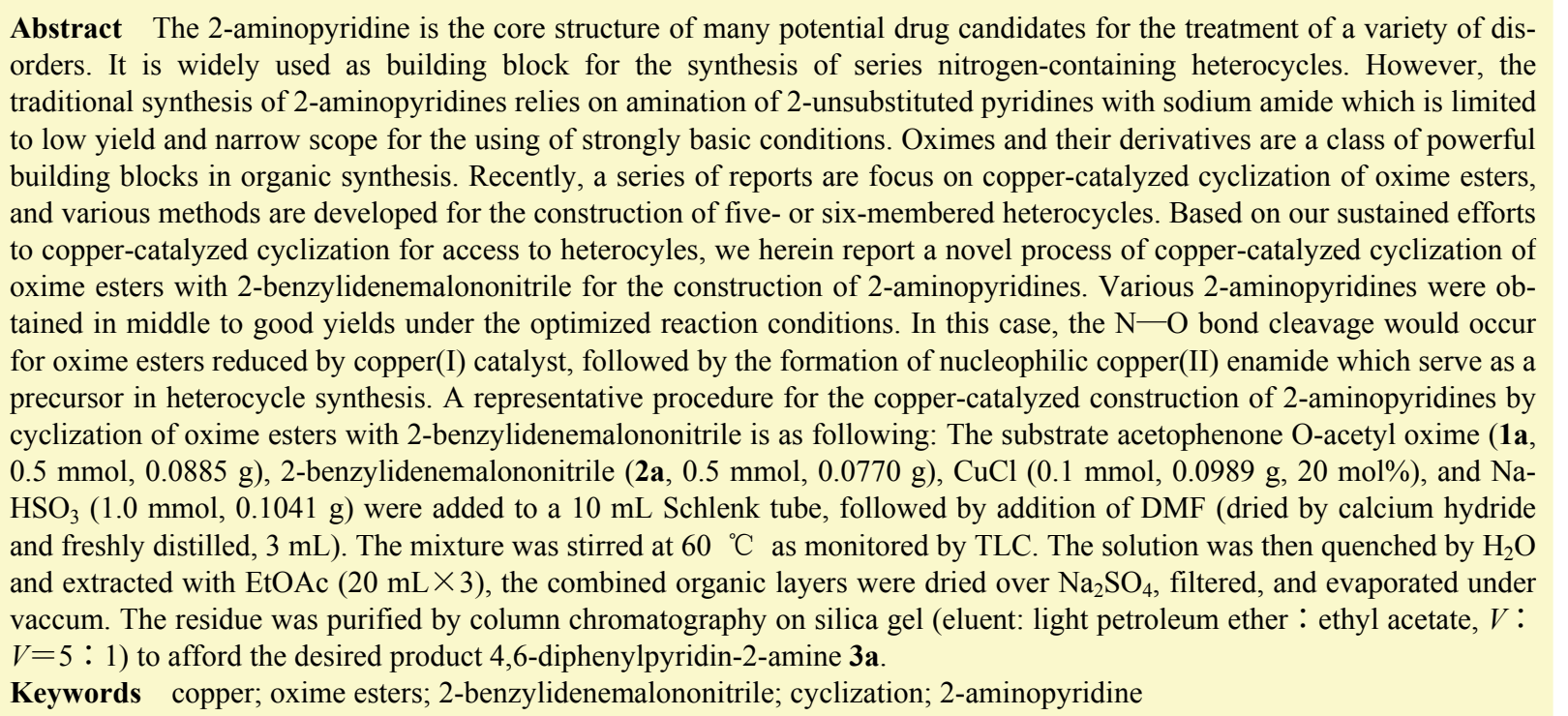 \\
\hline
\end{tabular}

\section{1 引言}

肜的衍生物是一类很有用的合成砌块, 在有机合成 中占有重要的地位 ${ }^{[1]}$. 通过该类化合物的贝克曼重排反 应可以很方便的构建一系列的酰胺产物 ${ }^{[2]}$, 也可利用该 类化合物的脱氢反应来制备腈类化合物 ${ }^{[3]}$. 最近, 铜催 化的肪的衍生物的环化反应引起了许多有机化学工作 者的关注, 一些通过铜催化的肟的衍生物的环化反应来 构建不同的五元或者六元杂环的方法见诸报道 ${ }^{[4]}$. 例如
西北大学关正辉老师课题组曾报道了一种新型的通过 铜催化条件下肜的衍生物与醛的偶联反应构建多取代 吡啶的方法(Figure 1, Eq. 1) ${ }^{[5 \mathrm{a}]}$. 最近，该课题组又发现， 在铜催化条件下, 苯乙酮肟的衍生物可以发生自偶联反 应，从而合成一系列对称的吡咯衍生物(Figure 1, Eq. $2)^{[5 b]}$. 与此同时, 华南理工大学的江焕峰老师课题组报 道了一种铜催化条件下，苯乙酮肟的衍生物与炔酯的 $[3$ +2]环化反应, 并成功构建了一系列的多取代吡咯产物 (Figure 1, Eq. 3) ${ }^{[6 \mathrm{a}]}$. 紧接着, 他们又发展了一种铜催化

\footnotetext{
*E-mail: shunyi@suda.edu.cn; shunjun@suda.edu.cn

Received April 9, 2014; published June 5, 2014.

Supporting information for this article is available free of charge via the Internet at http://sioc-journal.cn.

Project supported by Natural Science Foundation of China (No. 21172162), Young National Natural Science Foundation of China (No. 21202111) and Young Natural Science Foundation of Jiangsu Province (No. BK2012174).

项目受国家自然科学基金(No. 21172162)、国家青年自然科学基金(No. 21202111)及江苏省青年自然科学基金(No. BK2012174)资助.
} 
的苯乙酮肜与吡啶的氧化脱氢反应构建吡啶并咪唑类 衍生物的方法, 该方法具有简单、高效、对环境友好等 优点(Figure 1, Eq. 4) ${ }^{[66]}$. 最近, 该课题组对铜催化条件 下苯乙酮肜的偶联反应的研究又取得突破性的进展, 他 们发现在铜催化条件下, 苯乙酮肜可以和对甲苯亚磺酸 钠发生氧化偶联反应, 生成一系列的磺酰基乙烯胺类产 物, 该类产物很少见诸报道, 并且该类化合物可以进一 步水解形成一系列的砜类衍生物(Figure 1, Eq. 5) ${ }^{[6 \mathrm{cc}]}$. 新 加坡南洋理工大学的 Yoshikai 教授也对苯乙酮肜的反 应进行了一系列深入的研究, 他们通过铜催化条件下苯 乙酮肜的衍生物与肉桂醛的 $[3+3]$ 环化反应成功构建了

\section{Guan's work}<smiles>[R][R]1ccc2c(c1)C(=NO[C+])CC2</smiles>

一系列的多取代吡啶衍生物(Figure 1, Eq. 6) ${ }^{[7]}$. 通过对 一系列的铜催化的肜的衍生物的反应进行对比研究, 发 现在一价铜的催化还原作用下，肟的衍生物很容易发生 $\mathrm{N}-\mathrm{O}$ 键的断裂, 并进一步生成一种铜的络合物, 该络 合物很容易转换成烯胺结构, 具有一定的亲核性, 进而 发生一系列的加成或偶联反应.

2-氨基吡啶是很多药物分子的核心骨架，例如，抗 艾滋病 ${ }^{[8]}$ 、抗癌症 ${ }^{[9]}$ 、抗炎 ${ }^{[10]}$ 和抗神经性退行病 ${ }^{[11]}$ 的备 选药物当中均含有 2-氨基吡啶骨架. 而且, 2-氨基吡啶 还是构建很多复杂的含氮杂环化合物的起始原料, 例如

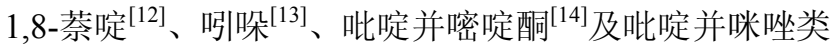<smiles>[R]c1ccc(C(CC)=NOC(C)=O)cc1</smiles>

\section{$\underset{\mathrm{NaHSO}_{3}, \mathrm{DMSO}}{\stackrel{\mathrm{CuBr}(10 \mathrm{~mol} \%)}{\longrightarrow}}$}

$120^{\circ} \mathrm{C}$

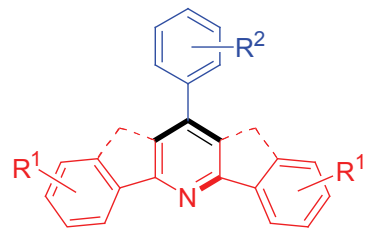

$120^{\circ} \mathrm{C}$<smiles>[R]c1ccc(-c2[nH]c(-c3ccc([R])cc3)c(C)c2C)cc1</smiles>

\section{Jiang's work}<smiles>[R]C#C[R]</smiles>

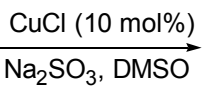
$120^{\circ} \mathrm{C}$<smiles></smiles><smiles>[R]C/C(=N\O[CH2+])c1cc[R1]cc1</smiles>

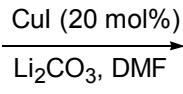
$95^{\circ} \mathrm{C}$<smiles>[R]c1ccc(-c2nc3ccccn3c2[R])cc1</smiles><smiles>[R]CC(=NOC(C)=O)c1cc#[R]cc1</smiles><smiles>[R]c1ccc(C(N)=C[Hg])cc1</smiles>

\section{Yoshikai's work}<smiles>[R]CC(=NOC(C)=O)c1cc#[R]cc1</smiles><smiles></smiles>

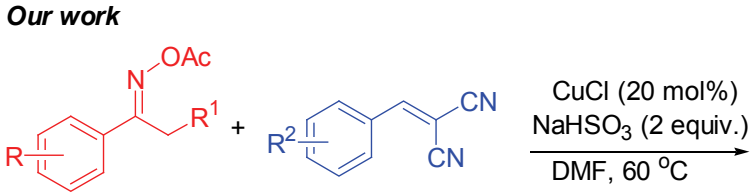<smiles>[R]c1cccc(-c2nc(N)c(C#N)c(-c3cccc[R]3[H])c2[R])c1</smiles>

图 1 铜催化的酮胿的反应

Figure 1 Copper-catalyzed cyclization of oxime esters 
化合物 ${ }^{[15]}$ 的合成都可以以 2-氨基吡啶为起始原料. 经典 的制备 2-氨基吡啶的方法是通过氨基钠对吡啶的氨化 反应(Chichibabin 反应)来制备, 但是该方法由于要用到 强碱, 所以导致底物的普适性和收率都不是很好 ${ }^{[16]}$. 我 们课题组也一直致力于铜催化的环化反应构建含氮杂 环化合物的研究, 并取得一定的进展 ${ }^{[17]}$, 作为工作的延 续, 我们也尝试着设计一些新型的铜催化的苯乙酮的肜 的衍生物的反应. 首先, 我们利用醛与丙二腈的缩合产 物苠烯丙二腈与乙酰基苯乙酮肟, 在溴化亚铜的催化作 用下进行反应. 令我们惊喜的是, 我们成功分离到了我 们预想的多取代 2-氨基吡啶产物(Figure 1, Eq. 7). 在我 们将这部分工作准备投稿时, 浙江大学崔孙良教授在 Org. Lett. 杂志上报道了铜催化新戊基肜的多组分反应 构建 2-氨基吡啶类化合物的方法 ${ }^{[18]}$.

\section{2 结果与讨论}

\section{1 反应条件的优化}

我们首先尝试了利用 1 equiv.乙酰基苯乙酮肜(1a) 和 1 equiv. 苠烯丙二腈(2a)以 $20 \mathrm{~mol} \% \mathrm{CuBr}$ 为催化剂, 2 equiv. 亚硫酸氢钠为添加剂, $3 \mathrm{~mL} \mathrm{DMF}$ 为溶剂条件下, $40{ }^{\circ} \mathrm{C}$ 搅拌 $8 \mathrm{~h}$. 令我们惊喜的是, 我们可以以 $26 \%$ 的液 相收率获得目标产物 2-氨基-4,6-二苯基吡啶 3a. 为了进 一步提高反应收率, 我们对不同的催化剂、溶剂、反应 温度以及添加剂做了进一步的笁选 (详细内容见 Supporting Information). 部分条件耖选结果列于表 1 当 中, 当我们将反应温度升高到 $60{ }^{\circ} \mathrm{C}$, 反应的收率可以 提高到 52\% (Table 1, Entry 2). 然而, 当我们继续升高反 应的温度时, 反应的收率并没有明显的提高(Table 1 , Entry 3). 接下来, 我们又对一系列的铜盐做了篮选 (Table 1, Entries 4 10), 发现氯化亚铜的催化效果最好, 可以 $74 \%$ 液相收率获得目标产物. $\mathrm{CuBr}_{2}$ 和 $\mathrm{CuCl}_{2}$ 对反应 基本没有催化效果(Table 1, Entries 8,9), 但是 $\mathrm{Cu}(\mathrm{OAc})_{2}$ 却能促进该反应的进行(Table 1, Entry 10). 我们也尝试 了一些其他的过渡金属催化剂, 如 $\mathrm{Fe}(\mathrm{III}), \mathrm{Co}(\mathrm{II})$ 和 $\operatorname{Ag}(\mathrm{I})$, 但是它们对该反应没有催化效果(Table 1, Entries $11 \sim 13)$. 当我们将反应置于纯的氩气或者氧气氛围当 中时, 反应的收率都没有得到提升(Table 1, Entries 14, 15). 但当我们用没有干燥过的 DMF 做溶剂时, 反应效 果很差(Table 1, Entry 16). 因此, 我们得出最优的反应 条件是: $1 \mathrm{a}(0.5 \mathrm{mmol}), 2 \mathrm{a}(0.5 \mathrm{mmol}), \mathrm{CuCl}(0.1 \mathrm{mmol})$, $\mathrm{NaHSO}_{3}(1.0 \mathrm{mmol})$, 无水 $\mathrm{DMF}(3 \mathrm{~mL}), 60{ }^{\circ} \mathrm{C}$ 反应 $8 \mathrm{~h}$.

\section{2 底物普适性的考察}

为了考证该反应对反应底物的普适性, 我们以一系 列不同的酮肜和芐烯丙二腈进行了反应. 正如 Table 2 中所列出的数据, 该反应可以中等到中等偏上的收率来 合成一系列的 2-氨基吡啶类化合物. 无论苠烯丙二腈的 芳环上有给电子基团, 卤素, 甚至是羟基取代, 该反应
表 1 反应条件的优化

Table 1 Optimization of the reaction conditions
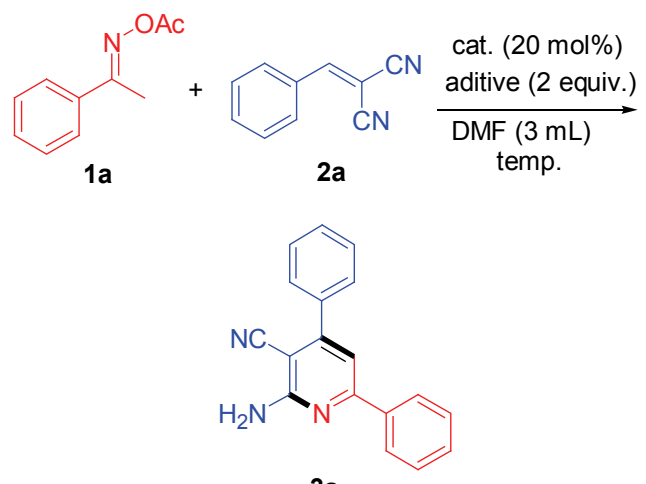

3a

\begin{tabular}{|c|c|c|c|c|c|}
\hline \multicolumn{2}{|c|}{ Entry Cat./mol\% } & \multicolumn{2}{|c|}{ Temp. $/ /^{\circ} \mathrm{C}$ Solvent $/ \mathrm{mL}$} & \multirow{2}{*}{$\begin{array}{l}\text { Additive/equiv. } \\
\mathrm{NaHSO}_{3}(2)\end{array}$} & \multirow{2}{*}{$\begin{array}{l}\text { Yield }^{a} / \\
(\mathrm{LC}-\mathrm{MS}) \\
26\end{array}$} \\
\hline 1 & $\mathrm{CuBr}(20)$ & 40 & DMF (3) & & \\
\hline 2 & $\mathrm{CuBr}(20)$ & 60 & DMF (3) & $\mathrm{NaHSO}_{3}(2)$ & 52 \\
\hline 3 & $\mathrm{CuBr}(20)$ & 80 & DMF (3) & $\mathrm{NaHSO}_{3}(2)$ & 37 \\
\hline 4 & $\mathrm{CuI}(20)$ & 60 & DMF (3) & $\mathrm{NaHSO}_{3}(2)$ & 37 \\
\hline 5 & $\mathrm{CuCl}(20)$ & 60 & DMF (3) & $\mathrm{NaHSO}_{3}(2)$ & $74(68)^{b}$ \\
\hline 6 & $\mathrm{Cu}_{2} \mathrm{O}(20)$ & 60 & DMF (3) & $\mathrm{NaHSO}_{3}(2)$ & 72 \\
\hline 7 & $\mathrm{Cu}(20)$ & 60 & DMF (3) & $\mathrm{NaHSO}_{3}(2)$ & 67 \\
\hline 8 & $\mathrm{CuBr}_{2}(20)$ & 60 & DMF (3) & $\mathrm{NaHSO}_{3}(2)$ & Trace \\
\hline 9 & $\mathrm{CuCl}_{2}(20)$ & 60 & DMF (3) & $\mathrm{NaHSO}_{3}(2)$ & Trace \\
\hline 10 & $\mathrm{Cu}(\mathrm{OAc})_{2}(20)$ & 60 & $\operatorname{DMF}(3)$ & $\mathrm{NaHSO}_{3}(2)$ & 67 \\
\hline 11 & $\mathrm{FeCl}_{3}(20)$ & 60 & DMF (3) & $\mathrm{NaHSO}_{3}(2)$ & NR \\
\hline 12 & $\mathrm{CoCl}_{2} \cdot 6 \mathrm{H}_{2} \mathrm{O}(20)$ & 60 & $\operatorname{DMF}(3)$ & $\mathrm{NaHSO}_{3}(2)$ & NR \\
\hline 13 & $\mathrm{AgNO}_{3}(20)$ & 60 & DMF (3) & $\mathrm{NaHSO}_{3}(2)$ & NR \\
\hline 14 & $\mathrm{CuCl}(20)$ & 60 & DMF (3) & $\mathrm{NaHSO}_{3}(2)$ & $51^{c}$ \\
\hline 15 & $\mathrm{CuCl}(20)$ & 60 & DMF (3) & $\mathrm{NaHSO}_{3}(2)$ & $35^{d}$ \\
\hline 16 & $\mathrm{CuCl}(20)$ & 60 & DMF (3) & $\mathrm{NaHSO}_{3}(2)$ & $27^{e}$ \\
\hline
\end{tabular}

${ }^{a}$ Reaction conditions: 1a $(0.5 \mathrm{mmol}), \mathbf{2 a}(0.5 \mathrm{mmol})$, cat. $(0.1 \mathrm{mmol}), \mathrm{NaHSO}_{3}$ $(1.0 \mathrm{mmol}), \mathrm{DMF}$ (dried by calcium hydride and freshly distilled, $3 \mathrm{~mL})$; the yields were determined by LC analysis using biphenyl as the internal standard; ${ }^{b}$ isolated yield; ${ }^{c}$ in the atmosphere of $\mathrm{Ar} ;{ }^{d}$ in the atmosphere of $\mathrm{O}_{2} ;{ }^{e} \mathrm{DMF}$ was used without purification.

都可以顺利的发生，并以较好的收率获得目标产物 $(3 \mathrm{~d} \sim 3 \mathrm{~g})$. $\alpha$-菜醛和 $\beta$-䒬醛合成的芐烯丙二腈也可以分 别以 $64 \%$ 和 $68 \%$ 的分离收率得到相应的目标产物(3h, 3i). 紧接着, 我们对一些多取代的芐烯丙二腈进行了考 察，如 2,4-二氯苯甲醛、胡椒醛、2,3,4-三甲氧基苯甲醛 做成的芐烯丙二腈, 均可以中等到中等偏上的收率获得 目标产物(3j～31，51\%～64\%). 而且，杂芳醛(2-噻吩醛) 做成的苠烯丙二腈也能进行该反应 $(3 \mathrm{~m}, 58 \%)$. 接着, 我 们又考察了不同取代的苯乙酮肜对反应的影响，首先， 我们考察了具有拉电子效应的取代基对反应的影响, 氟、氯、溴，甚至三氟甲基取代的苯乙酮肜都能顺利的 发生环化反应，并以 $38 \%$ ～64\%的分离收率获得目标产 物(3n 3p). 具有给电子效应的甲氧基和甲基苯乙酩肜 
表 2 底物的普适性考察

Table 2 Substrate scope of reaction<smiles>[R17]C/C(=N\O[R4](=[R])[H])c1cccc([R])c1</smiles><smiles>N#Cc1c(-c2ccccc2)cc(-c2ccccc2)nc1N</smiles>

3a, $68 \%$<smiles>N#Cc1c(-c2ccc(Cl)cc2)cc(-c2ccccc2)nc1N</smiles>

$3 f, 62 \%$<smiles>N#Cc1c(-c2ccc3c(c2)OCO3)cc(-c2ccccc2)nc1N</smiles>

3k, $51 \%$<smiles>N#Cc1c(-c2ccccc2)cc(-c2ccc(C(F)(F)F)cc2)nc1N</smiles>

$3 p, 52 \%$<smiles>N#Cc1c(N)nc2c(c1-c1ccccc1)CCc1ccccc1-2</smiles>

$3 u, 77 \%$

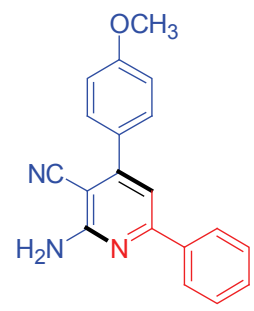

3b, $63 \%$<smiles>N#Cc1c(-c2ccc(O)cc2)cc(-c2ccccc2)nc1N</smiles>

3g, $63 \%$<smiles>COc1ccc(-c2cc(-c3ccccc3)nc(N)c2C#N)c(OC)c1OC</smiles>

3I, $64 \%$<smiles>N#Cc1c(-c2ccccc2)cc(-c2ccc(O)cc2)nc1N</smiles>

$3 q, 61 \%$<smiles>N#Cc1c(N)nc(-c2ccccc2)c(-c2ccccc2)c1-c1ccccc1</smiles>

$3 v, 31 \%$<smiles>Cc1ccc(-c2cc(-c3ccccc3)nc(N)c2C#N)cc1</smiles>

3c, $60 \%$<smiles>N#Cc1c(-c2cccc3ccccc23)cc(-c2ccccc2)nc1N</smiles>

3h, $64 \%$<smiles>N#Cc1c(-c2cccs2)cc(-c2ccccc2)nc1N</smiles>

$3 \mathrm{~m}, 58 \%$<smiles>Cc1ccc(-c2cc(-c3ccccc3)c(C#N)c(N)n2)cc1</smiles>

$3 r, 66 \%$<smiles>Cc1c(-c2ccccc2)nc(N)c(C#N)c1-c1ccccc1</smiles>

$3 \mathbf{w}, 81 \%$<smiles>N#Cc1c(-c2ccc(Br)cc2)cc(-c2ccccc2)nc1N</smiles>

3d, $69 \%$<smiles>N#Cc1c(-c2ccc3ccccc3c2)cc(-c2ccccc2)nc1N</smiles>

$3 i, 68 \%$<smiles>N#Cc1c(-c2ccccc2)cc(-c2ccc(Cl)cc2)nc1N</smiles>

3n, $51 \%$<smiles>Cc1ccccc1-c1cc(-c2ccccc2)c(C#N)c(N)n1</smiles>

3s, $57 \%$<smiles>CCc1c(-c2ccccc2)nc(N)c(C#N)c1-c1ccccc1</smiles>

$3 \mathbf{x}, 80 \%$<smiles>N#Cc1c(-c2ccc(F)cc2)cc(-c2ccccc2)nc1N</smiles>

3e, $51 \%$<smiles>N#Cc1c(-c2ccc(Cl)cc2Cl)cc(-c2ccccc2)nc1N</smiles>

$3 \mathbf{j}, 54 \%$<smiles>N#Cc1c(-c2ccccc2)cc(-c2ccc(Br)cc2)nc1N</smiles>

3o, $38 \%$

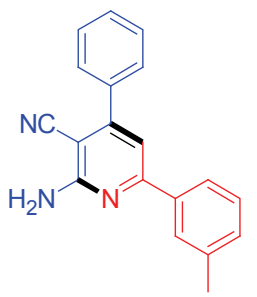

3t, $66 \%$

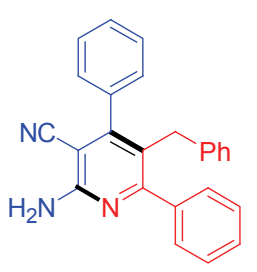

$3 y, 73 \%$

Reaction conditions: $1(0.5 \mathrm{mmol}), 2(0.5 \mathrm{mmol}), \mathrm{CuCl}(0.1 \mathrm{mmol}), \mathrm{NaHSO}_{3}(1.0 \mathrm{mmol})$, DMF (dried by calcium hydride and freshly distilled, $\left.3 \mathrm{~mL}\right), 60{ }^{\circ} \mathrm{C}, 8 \mathrm{~h}$.

可以分别以 $61 \%$ 和 $66 \%$ 的分离收率得到目标产物 $3 \mathbf{q}$ 和 3r. 而且，当甲基处于邻位或者间位时，该反应也能顺 利进行, 并与对位相当的收率得到相应的产物(3s, 57\%, 3t，66\%). 有趣的是，当我们将 $\alpha$-取代的苯乙酮肟(如苯

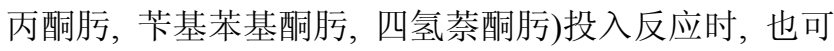
以取得较好的反应效果 $(3 \mathbf{u} \sim 3 \mathbf{w}, 31 \% \sim 81 \%)$. 


\section{3 可能的反应机理和结论}

基于以上结果及已有的文献报道 ${ }^{[5 \sim 7]}$, 我们对铜催 化的苯乙酮肜的环化反应提出了一个可能的反应机理 (Figure 2). 首先, 在铜(I)催化作用下, 苯乙酮肟发生 $\mathrm{N}-\mathrm{O}$ 键断裂形成一个亚胺自由基 $\mathbf{A}^{[19]}, \mathbf{A}$ 快速与铜络合 形成亚胺铜的络合物 $\mathbf{B}$, 接着 $\mathbf{B}$ 转换成具有亲核活性的 烯胺铜的络合物 $\mathbf{C}, \mathbf{C}$ 与芐烯丙二腈发生亲核加成反应 生成相应的中间体 $\mathbf{D}$, 紧接着发生分子内的亲核加成环 化得到中间体 $\mathbf{E}, \mathbf{E}$ 被进一步氧化成中间体 $\mathbf{F}$, 然后再异 构化生成相应的目标产物 3a. 同时, 还原剂 $\mathrm{NaHSO}_{3}$ 将 二价铜还原成一价铜, 使催化剂得以循环 ${ }^{[19 \mathrm{~d}]}$.

总结, 我们发展了一种新的通过铜催化条件下苯乙 醖肜的衍生物与苠烯丙二腈的环化反应来构建 2-氨基 吡啶类衍生物的方法. 在该反应过程中, 亚硫酸氢钠做 为添加剂, 对反应有很好的促进作用. 整个反应是通过
铜盐的氧化还原实现的，具有较好的底物普适性和较高 的收率. 同时也为合成一系列具有 2-氨基吡啶骨架的生 物活性分子提供了一种可供选择的方法. 我们对于通过 铜催化的苯乙酮肜的衍生物的环化反应来构建其他有 用的含氮杂环化合物的方法也在进一步深入的研究.

\section{4 实验部分}

通过铜催化条件下苯乙酮肟的衍生物与茮烯丙二 腈的环化反应来构建 2-氨基吡啶类衍生物的实验方法: 干燥的反应试管中加入乙酰基苯乙酩肜 $(0.5 \mathrm{mmol})$, 苠 烯丙二腈 $(0.5 \mathrm{mmol})$, 氯化亚铜 $(0.1 \mathrm{mmol})$, 亚硫酸氢钠 $(1.0 \mathrm{mmol})$ 和干燥的 DMF $(3 \mathrm{~mL})$, 搅拌 $8 \mathrm{~h}$ 后, 加水淬灭 反应，然后用乙酸乙酯洗涤萃取 $(20 \mathrm{~mL} \times 3)$, 合并滤液, 减压浓缩后所得粗产物进行柱层析(石油醚：乙酸乙酯, $V: V=5: 1$ )分离纯化, 得到多取代的 2-氨基吡啶化合物.<smiles>CC(=O)ON=C(C)c1ccccc1</smiles>

$\mathrm{Cu}^{2+}$

A

B<smiles>N#CC1C(=N)N=C(c2ccccc2)CC1c1ccccc1</smiles>

E $\mathrm{Cu}^{2+}$
$[\mathrm{O}]$

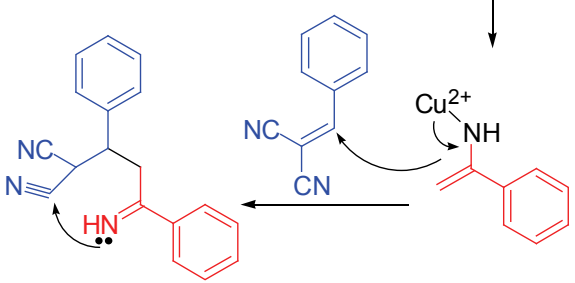

D

C

图 2 一种可能的反应机理

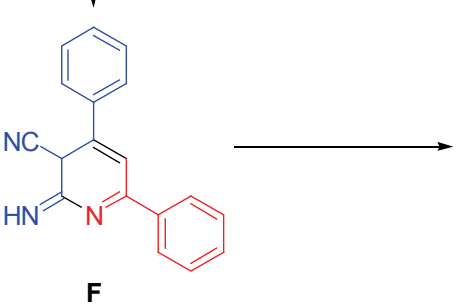<smiles>N#Cc1c(-c2ccccc2)cc(-c2ccccc2)nc1N</smiles>

3a

$\mathrm{Cu}^{2+} \stackrel{\mathrm{NaHSO}_{3}}{\longrightarrow} \mathrm{Cu}^{+}$

Figure 2 A plausible mechanism

\section{References}

[1] (a) Narasaka, K.; Kitamura, M. Eur. J. Org. Chem. 2005, 4505; (b) Himo, F.; Lovell, T.; Hilgraf, R.; Rostovtsev, V. V.; Noodleman, L.; Sharpless, K. B.; Fokin, V. V. J. Am. Chem. Soc. 2005, 127, 210; (c) Alonso, D. A.; Nájera, C. Chem. Soc. Rev. 2010, 39, 2891; (d) Sukhorukov, A. Y.; Ioffe, S. L. Chem. Rev. 2011, 111, 5004; (e) Cai, H.; Li, D.; Liu, Z.; Wang, G. Acta Chim. Sinica 2013, 71, 717. (蔡海婷, 李丹丹, 刘姿, 王官武, 化学学报, 2013, 71, 717); (f) Ran, L.; Liang, H.; Guan, Z. Chin. J. Org. Chem. 2013, 33, 66. (再陇飞, 梁浩,

关正辉，有机化学, 2013, 33, 66); (g) Dai, H.; Yu, H.; Liu, J.; Qin, X.; Wang, T.; Zhang, X.; Qin, Z.; Fang, J. Chin. J. Org. Chem. 2013, 33, 1104. (戴红, 手海波, 刘建兵, 秦雪, 王婷婷, 张欣, 秦振芳, 方建新, 有机化学, 2013, 33, 1104); (h) Liu, Z.; Wei, W.; Gan, C.; Huang, Y.; Liu, S.; Zhou, M.; Cui, J. Chin. J. Org. Chem. 2013, 33, 2551. (刘志平, 韦万兴, 甘春芳, 黄燕敏, 刘盛, 周敏, 崔建国, 有机化学, 2013, 33, 2551).

[2] (a) Ramalingan, C.; Park, Y.-T. J. Org. Chem. 2007, 72, 4536; (b) Hashimoto, M.; Obora, Y.; Sakaguchi, S.; Ishii, Y. J. Org. Chem. 2008, 73, 2894; (c) Ramón, R. S.; Bosson, J.; Díez-González, S.; 
Marion, N.; Nolan, S. P. J. Org. Chem. 2010, 75, 1197.

[3] (a) Choi, E.; Lee, C.; Na, Y.; Chang, S. Org. Lett. 2002, 4, 2369; (b) De Luca, L.; Giacomelli, G.; Porcheddu, A. J. Org. Chem. 2002, 67, 6272; (c) Augustine, J. K.; Atta, R. N.; Ramappa, B. K.; Boodappa, C. Synlett 2009, 3378; (d) Zhu, J.-L.; Lee, F.-Y.; Wu, J.-D.; Kuo, C.-W.; Shia, K.-S. Synlett 2007, 1317.

[4] (a) Tanaka, K.; Kitamura, M.; Narasaka, K. Bull. Chem. Soc. Jpn. 2005, 78, 1659; (b) Liu, S.; Yu, Y.; Libebeskind, L. S. Org. Lett. 2007, 9, 1947; (c) Liu, S.; Liebeskind, L. S. J. Am. Chem. Soc. 2008, 130, 6918; (e) Too, P. C.; Chua, S. H.; Wong, S. H.; Chiba, S. J. Org. Chem. 2011, 76, 6159.

[5] (a) Ren, Z.-H.; Zhang, Z.-Y.; Yang, B.-Q.; Wang, Y.-Y.; Guan, Z.-H. Org. Lett. 2011, 13, 5394; (b) Ran, R.; Ren, Z.-H.; Wang, Y.-Y.; Guan, Z.-H. Green Chem. 2014, 16, 112.

[6] (a) Tang, X.; Huang, L.; Qi, C.; Wu, W.; Jiang, H. Chem. Commun. 2013, 49, 9597; (b) Huang, H.; Ji, X.; Tang, X.; Zhang, M.; Li, X.; Jiang, H. Org. Lett. 2013, 15, 6254; (c) Tang, X.; Huang, L.; Xu, Y.; Yang, J.; Wu, W.; Jiang, H. Angew. Chem., Int. Ed. DOI: 10.1002/anie.201311217.

[7] Wei, Y.; Yoshikai, N. J. Am. Chem. Soc. 2013, 135, 3756.

[8] (a) Deng, J.; Sanchez, T.; Al-Mawsawi, L. Q.; Dayam, R.; Yunes, R. A.; Garofalo, A.; Bolger, M. B.; Neamati, N. Bioorg. Med. Chem. 2007, 15, 4985; (b) Fraley, A. W.; Chen, D.; Johnson, K.; McLaughlin, L. W. J. Am. Chem. Soc. 2003, 125, 616.

[9] Erić, S.; Ke, S.; Barata, T.; Solmajer, T.; Stanković, J. A.; Juranić, Z.; Savića, V.; Zloh, M. Bioorg. Med. Chem. 2012, 20, 5220.

[10] Laufer, S. A.; Zimmermann, W.; Ruff, K. J. J. Med. Chem. 2004, 47, 6311.
[11] Ji, H.; Delker, S. L.; Li, H.; Martásek, P.; Roman, L. J.; Poulos, T. L.; Silverman, R. B. J. Med. Chem. 2010, 53, 7804.

[12] Zong, R.; Zhou, H.; Thummel, R. P. J. Org. Chem. 2008, 73, 4334.

[13] (a) Chen, J.; Pang, Q.; Sun, Y.; Li, X. J. Org. Chem. 2011, 76, 3523 (b) Chen, J.; Song, G.; Pan, C. L.; Li, X. Org. Lett. 2010, 12, 5426.

[14] Bentabed-Ababsa, G.; Ely, S. C. S.; Hesse, S.; Nassar, E.; Chevallier, F.; Nguyen, T. T.; Derdour, A.; Mongin, F. J. Org. Chem. 2010, 75,839 .

[15] (a) Hand, E. S.; Paudler, W. W. J. Org. Chem. 1978, 43, 2900; (b) Denora, N.; Laquintana, V.; Pisu, M. G.; Dore, R.; Murru, L.; Latrofa, A.; Trapani, G.; Sanna, E. J. Med. Chem. 2008, 51, 6876; (c) Yan, R.-L.; Yan, H.; Ma, C.; Ren, Z.-Y.; Gao, X.-A.; Huang, G.-S.; Liang, Y.-M. J. Org. Chem. 2012, 77, 2024; (d) Zeng, J. Y.; Tan, J.; Leow, M. L.; Liu, X.-W. Org. Lett. 2012, 14, 4386; (e) He, C.; Hao, J.; Xu, H.; Mo, Y.; Liu, H.; Han, J.; Lei, A. Chem. Commun. 2012, 48, 11073; (f) Santra, S.; Bagdi, A. K.; Majee, A.; Hajra, A. Adv. Synth. Catal. 2013, 355, 1065; (g) Bagdi, A. K.; Rahman, M.; Santra, S.; Majee, A.; Hajra, A. Adv. Synth. Catal. 2013, 355, 1741.

[16] Chichibabin, A. E.; Zeide, O. A. J. Russ. Phys. Chem. Soc. 1914, 46, 1216

[17] (a) Cai, Z.-J.; Wang, S. Y.; Ji, S.-J. Org. Lett. 2012, 14, 6068; (b) Cai, Z.-J.; Wang, S. Y.; Ji, S.-J. Adv. Synth. Catal. 2013, 355, 2686.

[18] Wu, Q.; Zhang, Y.; Cui, S. Org. Lett. 2014, 16, 1350.

[19] (a) Trost, B. M.; Rhee, Y. H. J. Am. Chem. Soc. 2002, 124, 2528; (b) Nitta, M.; Iino, Y. Bull. Chem. Soc. Jpn. 1986, 59, 2365; (c) Deeming, A. J.; Owen, D. W.; Powell, N. I. J. Organomet. Chem. 1990 398, 299; (d) Guan, Z.-H.; Zhang, Z.-Y.; Ren, Z.-H.; Wang, Y.-Y.; Zhang, X. J. Org. Chem. 2011, 76, 339. 